\title{
Sinus of Valsalva aneurysm after blunt chest trauma with complicated perforation by infective endocarditis
}

\section{Cheol Won Hyeon ${ }^{1}$, Sung-Ji Park ${ }^{1,2}$, So Ree Kim ${ }^{1}$, Eun Kyoung Kim ${ }^{1,2}$, Sung Mok Kim ${ }^{2,3}$, Sung-A Chang ${ }^{1,2}$, Sang-Chol Lee ${ }^{1,2}$, Kiick Sung ${ }^{4}$, Seung Woo Park ${ }^{1,2}$}

${ }^{1}$ Division of Cardiology, Department of Medicine, Samsung Medical Center, Sungkyunkwan University School of Medicine, Seoul, Korea

${ }^{2}$ Cardiovascular Imaging Center, Heart Vascular Stroke Institute, Samsung Medical Center, Sungkyunkwan University School of Medicine, Seoul, Korea

${ }^{3}$ Department of Radiology, Samsung Medical Center, Sungkyunkwan University School of Medicine, Seoul, Korea

${ }^{4}$ Department of Thorax Surgery, Heart Vascular Stroke Institute, Samsung Medical Center, Sungkyunkwan University School of Medicine, Seoul, Korea

Received: March 15, 2019

Revised: May 28, 2019

Accepted: May 30, 2019

Corresponding author:

Sung-Ji Park

Division of Cardiology, Department of Medicine, Cardiovascular Imaging Center, Heart Vascular Stroke Institute, Samsung Medical Center, Sungkyunkwan University School of Medicine, 81 Irwonro, Gangnam-gu, Seoul 06351, Korea

Tel: +82-2-3410-0887

E-mail: tyche.park@gmail.com

This is an Open Access article distributed under the terms of the Creative Commons Attribution Non-Commercial License (http:// creativecommons.org/licenses/ by-nc/4.0/)

\section{ABSTRACT}

Sinus of Valsalva aneurysm (SVA), is a rare disease that may occur not only secondary to congenital or systemic diseases, but can also be related to acquired diseases such as aortic dissection, infection, and trauma. We report a rare case of SVA that presented as fever. We made a fairly accurate diagnosis that aneurysmal perforation into the right ventricle from complicated infective endocarditis using multimodality imaging tools, including transthoracic, transesophageal echocardiography and computed tomography. With regard to the cause of SVA, there were no associated findings of congenital or systemic diseases. However, we have noticed that the patient had a critical motorcycle accident 4 years ago. Based on the medical records and images at that time, we could estimate that the blunt chest trauma was the possible cause. Here, we share exciting images obtained during the process of diagnosing the patient and identifying the mechanisms of this uncommon disease.

Keywords: Accidents, traffic; Aneurysm; Endocarditis; Fistula; Sinus of Valsalva

\section{INTRODUCTION}

Sinus of Valsalva aneurysm (SVA) is a disease in which the aortic root area increases between the aortic valve annulus and the sinotubular ridge [1]. Whether congenital or acquired, SVA results from the weakened elastic lamina at the junction of the aortic media and the annulus fibrosis [2]. It is a rare disease, with an estimated incidence in the general population of $0.09 \%$, comprising $0.1 \%$ to $3.5 \%$ of all congenital heart diseases [3]. 
We recently experienced a rare case of SVA presumed to be caused by blunt chest trauma 4 years prior that presented as fever from complicated infective endocarditis and aneurysmal perforation into the right ventricle. We made a fairly accurate diagnosis using multimodality imaging tools including transthoracic echocardiography (TTE), transesophageal echocardiography (TEE) and computed tomography (CT). Here we share exciting images obtained during the process of diagnosing the patient and identifying the mechanisms of this uncommon disease.

\section{CASE REPORT}

A 26-year-old man was admitted to our hospital after under-
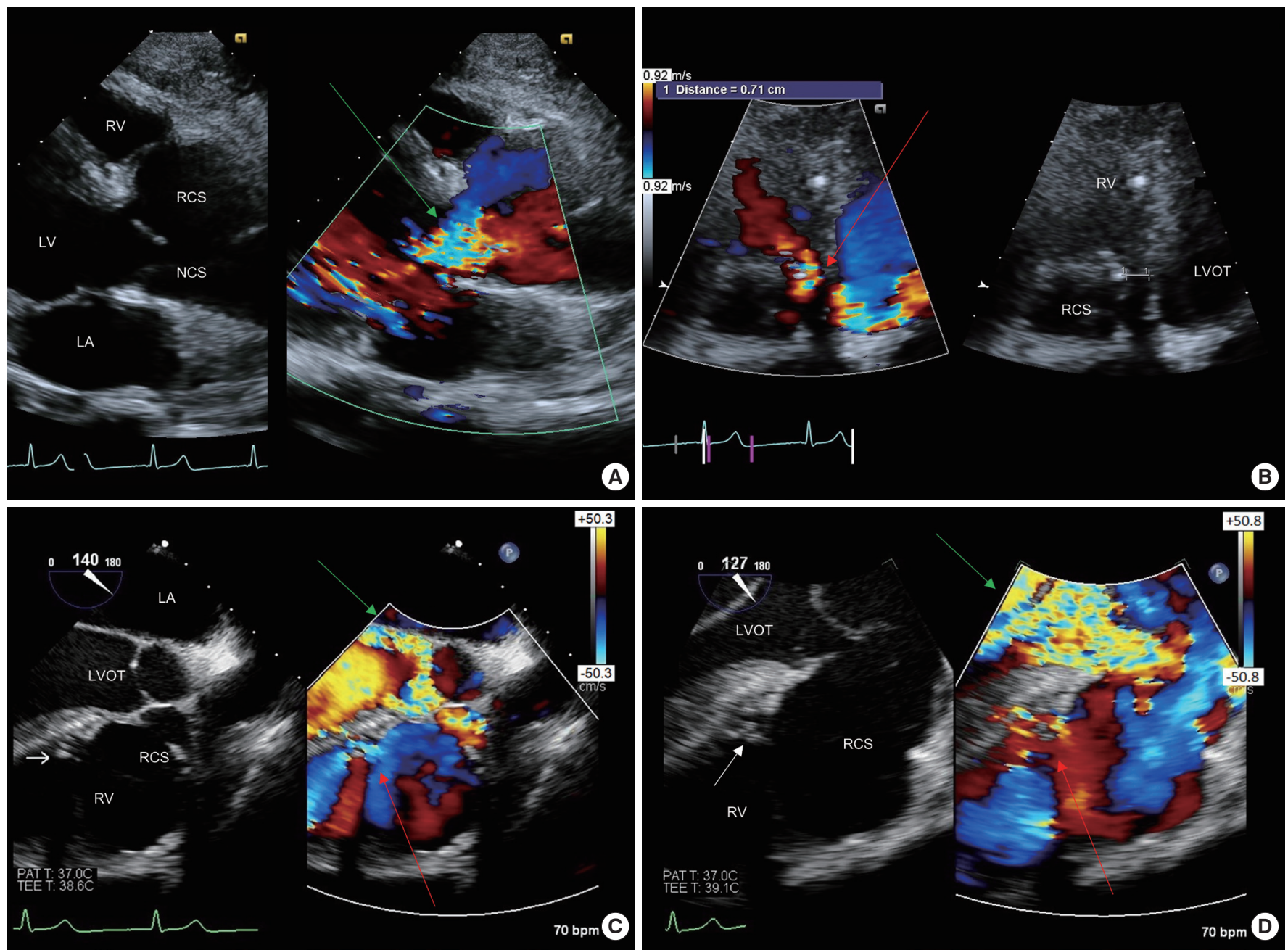

Fig. 1. Transthoracic echocardiography and transesophageal echocardiography (TEE). (A) Parasternal long-axis view showed dilated right coronary sinus (RCS) accompanying significant aortic regurgitation (AR) (green arrow). (B) On modified apical five-chamber view, a left-toright shunt flow (red arrow) exists from the dilated RCS to the right ventricle (RV). (C) On a $140^{\circ}$ view of the TEE, a shunt flow (red arrow) from the dilated RCS to RV (white arrow) accompanying significant AR (green arrow) is visible. (D) On a $127^{\circ}$ view of TEE, a $1.11 \times 0.37 \mathrm{~cm}$ sized oscillating material with a shunt flow (red arrow) is seen at the fistula (white arrow). AR flow was marked with green arrow. LA, left atrium; LV, left ventricle; NCS, non-coronary sinus; LVOT, left ventricular outflow tract. 
Upon visiting our hospital, the patient had no remarkable symptoms such as fever, chest pain, or dyspnea. There were also no signs of congestion such as pretibial pitting edema or jugular vein distention. A grade IV or higher diastolic murmur was auscultated at the right upper sternal border.

Laboratory tests showed the following normal findings: white blood cell count 5,620 cells $/ \mu \mathrm{L}$; hemoglobin $14.5 \mathrm{~g} / \mathrm{dL}$; platelets 159,000 cells/ $\mu \mathrm{L}$; and C-reactive protein $0.2 \mathrm{mg} / \mathrm{dL}$. Additional blood cultures at our hospital showed no growth of organisms. TTE revealed significant AR with a $5.2 \times 3.3 \mathrm{~cm}$ aneurysmal dilatation of the right coronary sinus (Fig. 1A). The right coronary sinus aneurysm dilated toward the right ventricle with an aneurysmal defect and color acceleration flow, indicating perforation and a left-to-right shunt via a fistula to the right ventricle (Fig. 1B). However, no definite vegetation was noted. On TEE, a $1.11 \times 0.37 \mathrm{~cm}$ area of oscillating material was visible at the fistula of the perforated SVA suggestive of vegetation (Fig. 1C, D). A positron emission tomography (PET) scan was performed to confirm acute infection, but no hot uptake was observed in the heart (Fig. 2A). On contrast $\mathrm{CT}$, the $85-\mathrm{mm}$ aneurysm and perforation into the right ventricle were confirmed (Fig. 2B).

In the surgical field, we noted fibrosis of the dilated right coronary cusp, suggesting healed infective endocarditis. The dissection flap was located on the right coronary sinus and the false lumen was a pseudoaneurysm, forming a 3-mm fistula toward the anterior wall of the right ventricle (the dissection flap was not clearly identified in any preoperative images). The longer diameter of the total aneurysm was about $8 \mathrm{~cm}$. The perforated right coronary cusp was repaired using fresh autologous pericardium. The surgeon performed partial aortic root remodeling by placing a $26-\mathrm{mm}$ hemashield vascular graft at the ascending aorta. The perforated pseudoaneurysm of the right coronary sinus was sutured inward and connected with the graft, whereas the right coronary artery was re-implanted into the vascular graft by the creation of a button. The final pathologic finding of the resected aortic wall showed dissected adventitia with hemorrhage, consistent with pseudoaneurysm. Postoperatively, the patient recovered without any significant complications.

The patient had no history of congenital heart disease or risk factors of infective endocarditis such as intravenous drug abuse. To identify the cause of the aneurysm, a more detailed medical history was taken. He disclosed having suffered a traffic accident while on a motorcycle 4 years ago. We assumed that the physical impact was considerable due to
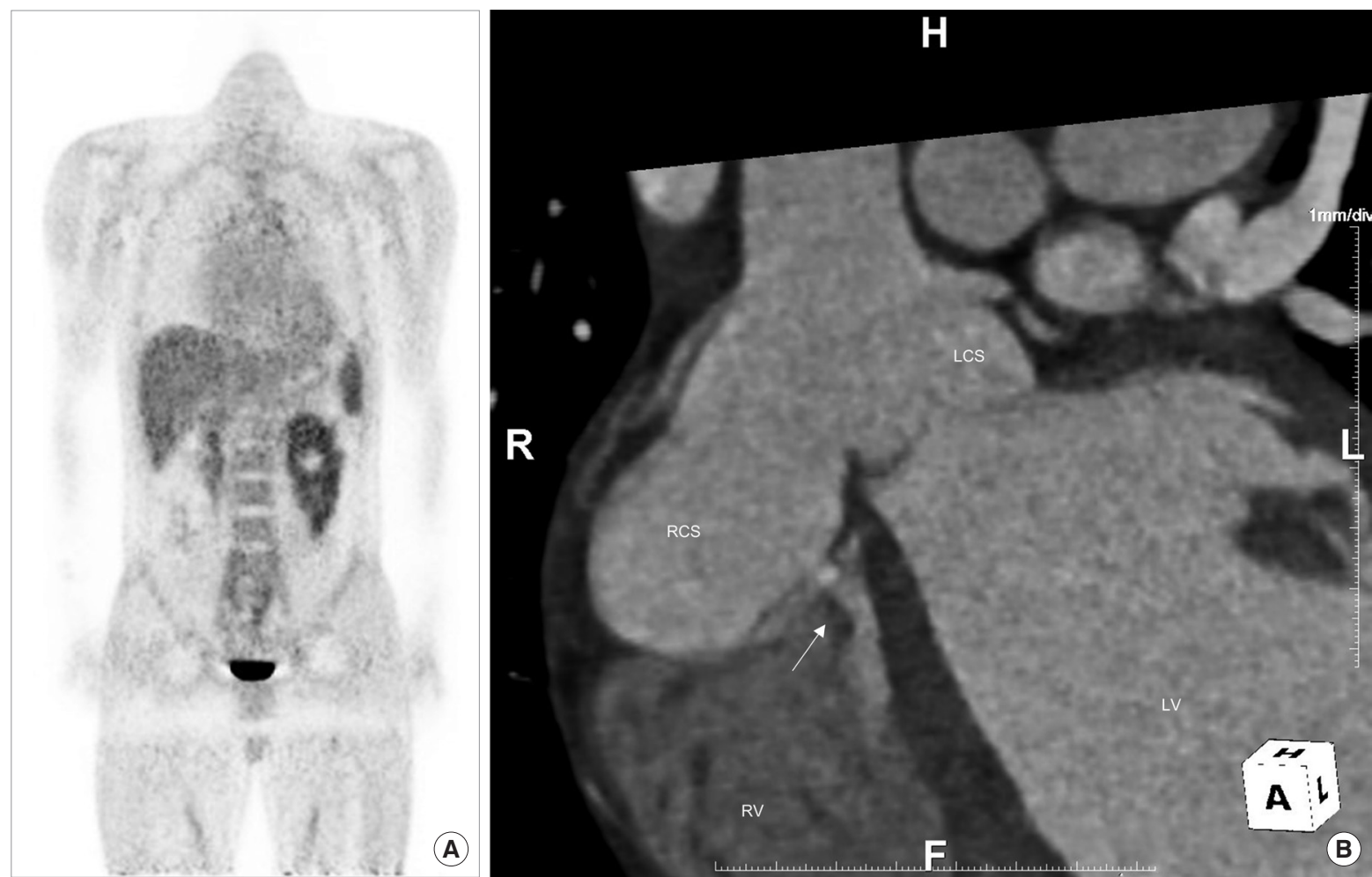

Fig. 2. (A) Positron emission tomography image. No hot uptake is observed. (B) Reconstructed contrast chest computed tomography image. Contrast leakage (arrow) from the right coronary sinus (RCS) into the right ventricle (RV) is observed. LV, left ventricle; LCS, left coronary sinus. 

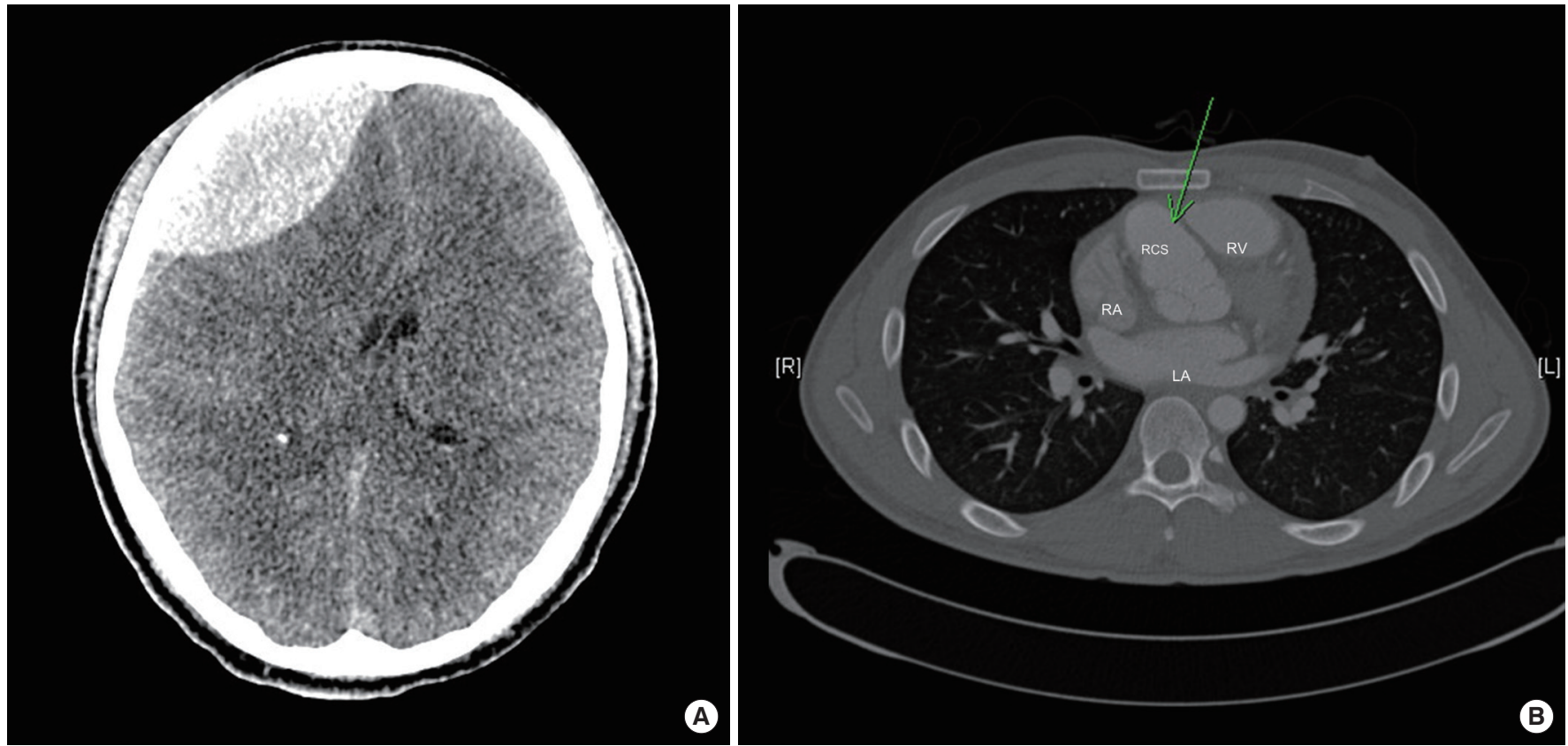

Fig. 3. (A) Non-contrast brain computed tomography (CT) image. An epidural hemorrhage is visible in the right frontal lobe accompanying the midline shift. (B) Contrast chest CT image. The dilated right coronary sinus (RCS) of Valsalva sinus (arrow) toward the right ventricle (RV) is observed as an unruptured state. LA, left atrium; RA, right atrium.

the fact that the patient suffered an epidural hemorrhage of the right frontal lobe with concomitant intracranial hemorrhage by head trauma at that time (Fig. 3A) for which he underwent surgical treatment and ventilator care. Although the patient had no specific thoracic symptoms, chest CT was performed to rule out intrathoracic injury induced by the blunt chest trauma 2 months after the accident. At that time, an unruptured Valsalva sinus aneurysm with small pericardial effusion was confirmed (Fig. 3B). However, the patient was unaware of the diagnosis and did not receive any follow-up care at that time.

The patient agreed to provide Samsung Medical Center with his personal information for research purposes.

\section{DISCUSSION}

SVA is usually congenital. It may be secondary from systemic disease such as cystic medial necrosis, Marfan's syndrome, and Behçet disease. However, it may be related to acquired disease, such as aortic dissection, infection, and trauma [1]. In particular, a decelerational thoracic injury from acute trau$\mathrm{ma}$ is also a major cause of aortic aneurysm and rupture. The definition of a decelerational thoracic injury is a complication of a sudden movement involving the thorax that strikes an obstacle and is stopped abruptly, causing different decel- eration rates of the fixed structures and disruption of the great vessels [4]. Injury of ascending aorta after a rapid deceleration is rare but so fatal that most of its course is death or an aortic rupture within 8 days [5]. However, a small number of the patients survive asymptomatically, and there has been a reported case who was asymptomatic for up to 41 years after the accident and presented as a rupture of SVA [6].

In this case, the presence of SVA was detected by a chest CT scan 2 months after the motorcycle traffic accident for the first time. It is impossible to determine whether the SVA in this case was congenital or caused by trauma completely. However, considering that congenital SVA is frequently associated with Marfan's syndrome, Ehlers-Danlos syndrome, or other connective tissue disorders [7], the likelihood of a congenital anomaly is low in this case since the patient had no relevant signs. Although congenital SVA mostly affects right coronary sinus, the deceleration force of motorcycle accident at that time is presumed to be directed toward anterior direction based on the estimated mechanism of accident and the presence of epidural hemorrhage in the right frontal lobe. Therefore, it is possible to presume that the blunt chest trauma may have affected right coronary sinus to be dilated toward the anterior side of the right ventricle.

No aneurysm perforation or shunt was evident in the CT images taken 4 years prior, so this perforation and shunt 
were likely due to infective endocarditis. Related to the absence of hot uptake on PET, the use of antibiotics for several weeks may have been the reason. Considering the vegetation in the fistula site and positive blood cultures before admission, it is not unreasonable to believe that the infective endocarditis directly caused the perforation. Factors that determine symptom severity are age at presentation, shunt size, and the presence of associated lesions [2]. Clinical presentation may range from asymptomatic to shortness of breath, chest pain, and possibly poor prognosis with a short life expectancy $[3,8]$. In this case, the patient complained of only fever and had no other remarkable symptoms such as dyspnea, orthopnea, or peripheral edema. We estimated that the perforation, shunt, and AR caused no significant hemodynamic changes. However, the operation is considered an appropriate treatment approach because an increasing associated AR or shunt may cause structural aggravation and finally worsen the symptoms and prognosis.

Detection of perforation and shunt through imaging studies such as TTE, TEE, and CT was crucial in the determination of a diagnosis and surgical treatment. The diagnostic accuracy of TTE was $75 \%$, while that of TEE was $90 \%$. The accuracy rates of $\mathrm{CT}$ and magnetic resonance imaging are even higher [9]. Multimodal imaging tools are used to evaluate the orientation of the surgical procedure and the involved sinus, severity, and the presence of shunt, concomitant cardiac or vascular abnormalities [9]. Although we failed to recognize the dissection flap before the operation, we made a fairly accurate diagnosis that aneurysmal perforation into the right ventricle from complicated infective endocarditis using multimodal imaging tools. Subsequently, surgical treatment could be determined. Therefore, multimodality imaging tools should be used appropriately when diagnosing SVA and perforation.

\section{CONFLICTS OF INTEREST}

No potential conflict of interest relevant to this article was reported.

\section{ORCID}

Cheol Won Hyeon https://orcid.org/0000-0003-3992-6364

Sung-Ji Park https://orcid.org/0000-0002-7075-847X

So Ree Kim https://orcid.org/0000-0002-3164-3258

Eun Kyoung Kim https://orcid.org/0000-0002-7653-3503

Sung Mok Kim https://orcid.org/0000-0001-5190-2328

Sung-A Chang https://orcid.org/0000-0001-5124-605X

Sang-Chol Lee https://orcid.org/0000-0003-2176-0482

Kiick Sung https://orcid.org/0000-0003-0768-9587

Seung Woo Park https://orcid.org/0000-0002-2941-515X

\section{REFERENCES}

1. Bricker AO, Avutu B, Mohammed TL, Williamson EE, Syed IS, Julsrud PR, et al. Valsalva sinus aneurysms: findings at CT and MR imaging. Radiographics 2010;30:99-110.

2. Moustafa S, Mookadam F, Cooper L, Adam G, Zehr K, Stulak J, et al. Sinus of Valsalva aneurysms: 47 years of a single center experience and systematic overview of published reports. Am J Cardiol 2007;99:1159-64.

3. Feldman DN, Roman MJ. Aneurysms of the sinuses of Valsalva. Cardiology 2006;106:73-81.

4. Swan KG Jr, Swan BC, Swan KG. Decelerational thoracic injury. J Trauma 2001;51:970-4.

5. Parmley LF, Mattingly TW, Manion WC, Jahnke EJ Jr. Nonpenetrating traumatic injury of the aorta. Circulation 1958;17:1086-101.

6. Greiss I, Ugolini P, Joyal M, Bouchard D, Mercier LA. Ruptured aneurysm of the left sinus of Valsalva discovered 41 years after a decelerational injury. J Am Soc Echocardiogr 2004;17:906-9.

7. Ott DA. Aneurysm of the sinus of Valsalva. Semin Thorac Cardiovasc Surg Pediatr Card Surg Annu 2006;9:165-76.

8. Wei J, Chang CY, Chang YC. Traumatic sinus of Valsalva fistula. Ann Thorac Surg 1991;52:852-4.

9. Galicia-Tornell MM, Marin-Solis B, Mercado-Astorga O, Espinoza-Anguiano S, Martinez-Martinez M, VillalpandoMendoza E. Sinus of Valsalva aneurysm with rupture. Case report and literature review. Cir Cir 2009;77:441-5. 